\title{
Mechanisms of poly(ADP-ribosyl)ation and its role in genotoxic stress response
}

\author{
A. Mangerich \\ Molecular Toxicology Group, University of Konstanz, Germany \\ e-mail: aswin.mangerich@uni-konstanz.de
}

Key words: Poly(ADP-ribosyl)ation, PARP1, DNA repair, p53, WRN, genotoxicity

\begin{abstract}
Motivation and Aim: PARP1 catalyzes the posttranslational protein modification with poly(ADP-ribose) (PAR) of variable chain length and branching frequency. Poly(ADPribosyl)ation (PARylation) is involved in diverse cellular functions, such as DNA repair, transcription, and regulation of cell death, and contributes to various pathophysiological conditions. Importantly, PARP inhibitors are being employed and further developed in clinical cancer therapy. The overall aim of our research interests is to unravel the biochemical and cellular functions of PARP1-mediated PARylation during genotoxic stress response.
\end{abstract}

Methods and Algorithms: We employ a broad spectrum of state-of the art bioanalytic, biochemical, molecular biological, bio-imaging, and genetic techniques, including quantitative isotope dilution mass spectrometry, gene expression and proteomics analyses, high-end microscopy and automated image analysis, and genetic engineering via CRISPR/Cas9.

Results: This talk will give a brief overview of our latest published results dealing with biochemical and cellular mechanisms of PARylation, including its role in the regulation of the tumor suppressor protein p53 [1-3]. In addition the relevance of PARylation in patho-physiological mechanisms in in vivo mouse models will be highlighted $[4,5]$. Furthermore, unpublished results will be presented.

Conclusion: PARylation represents a complex and versatile post-translational modification with key roles in genotoxic stress response on a cellular level and significant contributions to patho-physiological states on the organismic level. Thereby pharmacological interventions hold a high potential in terms of cancer treatment and other pathophysiological conditions.

Acknowledgements: Our work is supported by the German Research Foundation (DFG), the Konstanz Graduate School of Chemical Biology, and the Young Scholar Fund of University of Konstanz.

\section{References}

1. Rank L., Veith S., Gwosch E.C. et al. (2016) Analyzing structure-function relationships of artificial and cancer-associated PARP1 variants by reconstituting TALEN-generated HeLa PARP1 knock-out cells. Nucleic Acids Research. 44(21):10386-10405. DOI 10.1093/nar/gkw859.

2. Fischbach A., Kruger A., Hampp S. et al. (2018) The C-terminal domain of p53 orchestrates the interplay between non-covalent and covalent poly(ADP-ribosyl)ation of p53 by PARP1. Nucleic Acids Research. 46(2):804-822. DOI 10.1093/nar/gkx1205.

3. Fischer J.M.F., Zubel T., Jander K. et al. (2017) PARP1 protects from benzo[a]pyrene diol epoxideinduced replication stress and mutagenicity. Archives Toxicology. DOI 10.1007/s00204-017-2115-6.

4. Dörsam B., Seiwert N., Foersch S. et al. (2018) PARP-1 protects against colorectal tumor induction, but promotes inflammation-driven colorectal tumor progression. PNAS. DOI 10.1073/pnas.1712345115.

5. Schuhwerk H., Bruhn C., Siniuk K. et al. (2017) Kinetics of poly(ADP-ribosyl)ation, but not PARP1 itself, determines the cell fate in response to DNA damage in vitro and in vivo. Nucleic Acids Research. DOI $10.1093 / \mathrm{nar} / \mathrm{gkx} 717$. 